\title{
1 The impact of Spike mutations on SARS-CoV-2 neutralization
}

Rees-Spear $C^{1}$, Muir $L^{1}$, Griffith $S A^{1}$, Heaney $J^{2}$, Aldon $Y^{3}$, Snitselaar $L^{3}$, Thomas $P^{1}$, Graham $C^{4}$, Seow $J^{4}$, Lee $N^{1}$, Rosa $A^{5}$, Roustan $C^{5}$, Houlihan $C^{2,6}$, Sanders $R W^{3}$, Gupta $R^{8}$, Cherepanov ${ }^{5}$, Stauss $H^{1}$, Nastouli $E^{2,5,8}$ on behalf of the SAFER Investigators $\pi$, Doores $\mathrm{KJ}^{4}$, van Gils MJ', McCoy LE ${ }^{1 *}$

${ }^{1}$ Institute of Immunity and Transplantation, Division of Infection and Immunity, University College London, UK

${ }^{2}$ Advanced Pathogens Diagnostic Unit, Department of Clinical Virology, University College London

8 Hospitals NHS Foundation Trust, UK

9 32Department of Medical Microbiology, Academic Medical Center, University of Amsterdam,

10 Amsterdam Institute for Infection and Immunity, Netherlands

114 School of Immunology \& Microbial Sciences, King's College London, UK

12 5The Francis Crick Institute, UK

13 6Research Department of Infection, Division of Infection and Immunity, University College London

147 Department of Medicine, University of Cambridge, UK

$15{ }^{8}$ Great Ormond Street Institute for Child Health, Infection, Immunity and Inflammation, University

16 College London

$17 \pi$ TSAFER Investigators are listed in the supplementary information

18 *Correspondence: L.mccoy@ucl.ac.uk

\section{Abstract}

20 Multiple SARS-CoV-2 vaccines have shown protective efficacy, which is most likely mediated by

21 neutralizing antibodies recognizing the viral entry protein, Spike. Antibodies from SARS-CoV-2

22 infection neutralize the virus by focused targeting of Spike and there is limited serum cross-

23 neutralization of the closely-related SARS-CoV. As new SARS-CoV-2 variants are rapidly

24 emerging, exemplified by the B.1.1.7, 501Y.V2 and P.1 lineages, it is critical to understand if 25 antibody responses induced by infection with the original SARS-CoV-2 virus or the current 26 vaccines will remain effective against virus variants. In this study we evaluate neutralization of a series of mutated Spike pseudotypes including a B.1.1.7 Spike pseudotype. The analyses of a

28 panel of Spike-specific monoclonal antibodies revealed that the neutralizing activity of some 29 antibodies was dramatically reduced by Spike mutations. In contrast, polyclonal antibodies in 30 the serum of patients infected in early 2020 remained active against most mutated Spike 31 pseudotypes. The majority of serum samples were equally able to neutralize the B.1.1.7 Spike 32 pseudotype, however potency was reduced in a small number of samples (3 of 36) by 5-1033 fold. This work highlights that changes in the SARS-CoV-2 Spike can alter neutralization 34 sensitivity and underlines the need for effective real-time monitoring of emerging mutations and their impact on vaccine efficacy. 


\section{Introduction}

Serum neutralization activity is a common correlate of protection against viral infection following vaccination or natural infection (Plotkin, 2008). However, effective protection from viral infection can also require sufficient breadth of serum neutralization rather than potency alone. This is because of the high-levels of variation observed in major antigens across some viral populations (Burton, Poignard, Stanfield, \& Wilson, 2012). The classic example in which a lack of breadth limits the protective capacity of the antibody response is Influenza. Here, the majority of neutralizing serum antibodies target only a particular set of influenza strains as a result of antigenic drift of the immunodominant hemagglutinin head (Zost, Wu, Hensley, \& Wilson, 2019). Due to this, an annual vaccine is required and must be matched to the most probable circulating strain in any given year to ensure protection from infection. Emerging data from human vaccine trials and challenge studies in animal models suggest that neutralizing antibodies can prevent disease caused by infection with SARS-CoV-2, the virus that causes COVID-19 (McMahan et al., 2020; Polack et al., 2020). However, new variants of SARS-CoV-2 have begun to emerge in both human and farmed animal populations (S. Kemp et al., 2020; Oude Munnink et al., 2020; Tegally et al., 2020; Welkers, Han, Reusken, \& Eggink, 2020). These variants include mutations in the major neutralizing antigen, the Spike glycoprotein, and raises the question of whether neutralizing serum responses induced by early circulating strains or by vaccines based on the Spike sequence of these early strains can neutralize the recently emerged virus variants.

Prior to the emergence of multiple mutations in Spike in the human population, we reasoned that a logical way to identify potential escape mutations was to look at sites of amino acid variation relative to the most closely related human betacoronavirus SARS-CoV, which caused the original SARS outbreak in 2003 (CDC, 2003). These two closely related viruses are characterized by a notable difference in transmission dynamics and disease outcomes (Cevik, Kuppalli, Kindrachuk, \& Peiris, 2020; Lipsitch et al., 2003; Petersen et al., 2020) but both use the human ACE2 protein as a viral entry receptor (W. Li et al., 2003) and share approximately 75\% similarity overall in Spike at an amino acid level (Gralinski \& Menachery, 2020). Both viruses use the same region of their respective Spikes to bind ACE2; the receptor binding domain (RBD - found within the S1 subunit of Spike). There is considerable amino acid variation between the two RBDs, despite their conserved binding to ACE2, which explains why the majority of SARS-CoV-induced neutralizing monoclonal antibodies (mAbs) were found not to neutralize SARS-CoV-2, although some cross-binding activity has been observed (Wu et al., 2020) and targeted mutations can enable neutralization (Liu et al., 2020). Similarly, the 
majority of COVID-19 sera have either weaker or no neutralizing activity against SARS-CoV, but cross-neutralizing mAbs have been isolated (Brouwer et al., 2020).

Since the start of the pandemic, sequencing of viral populations has been deployed to enable detection of individual mutations within SARS-CoV-2 and identify new variants or strains that become dominant. Most recently, a new variant, B.1.1.7, has emerged in the UK (S. Kemp et al., 2020; Rambaut et al., 2020) that includes multiple mutations in both the RBD and the N-terminal domain (NTD) of Spike, both targets for neutralizing antibodies. Similarly, two further variants have been identified in South Africa, 501Y.V2, and Brazil, P.1, which carry additional mutations in both the NTD and RBD (Faria et al., 2021; Tegally et al., 2020). All three variants share a deletion of 3 amino acids in Orf1ab and key mutations in the RBD (E484K and the N501Y); data so far consistent with convergent evolution. Early reports have indicated that while the RBD mutation N501Y in the B.1.1.7 strain does not compromise post vaccine serum neutralization (Xie et al., 2021), one of the individual changes in the 501Y.V2 strain does impair neutralization but does not remove all activity (Greaney et al., 2021). Moreover, in vitro escape studies have shown similar mutations occur under selective pressure (Andreano et al., 2020).

Therefore, in this study we evaluated the potential role of individual amino acids in facilitating escape from neutralizing antibodies. Firstly, by making a series of point mutations to change the amino acids in SARS-CoV-2 to those found at the analogous position in SARS-CoV. Secondly, by making individual point mutations emerging in real world populations and by generating a pseudotype virus using the B.1.1.7 variant Spike sequence. We identify multiple mutations that can abrogate neutralization by some monoclonal antibodies targeting the RBD of Spike. However, in contrast, we show that serum responses are more resilient to these mutations, especially following severe infection where the antibody response is characterized by increased breadth.

\section{Results}

\section{Generation of potential escape mutants by SARS-CoV amino acids substitution}

There are 56 individual amino acid changes between the RBD of SARS-CoV-2 and SARS-CoV (Ortega, Serrano, Pujol, \& Rangel, 2020), including sites at which antibody escape has been observed for SARS-CoV (Rockx et al., 2010). We identified 15 sites where single, or sequential, non-conservative amino acid changes were observed compared to SARS-CoV. These sites were mutated in the SARS-CoV-2 Spike to match SARS-CoV (Fig S1). Mutated Spike protein plasmids were then co-transfected with a lentiviral construct encoding luciferase and a packaging plasmid to produce pseudotyped viruses (Seow et al., 2020). Three of the substitutions resulted in virus that did not give sufficient titer to evaluate the impact on neutralization 
104 activity. The remaining mutated pseudotypes were then screened for any alteration in 105 neutralization against a panel of human mAbs (Brouwer et al., 2020) isolated post SARS-CoV-2 106 infection. These mAbs have been previously mapped into eleven binding clusters whereby 107 mAbs within a cluster reciprocally compete for binding to Spike. Of these, eight clusters 108 comprise neutralizing mAbs (I, III, IV, VI, VII, IX, X, XI), five of which target the RBD (I, III, VI, VII, 109 IX), and three clusters contain only non-neutralizing mAbs (II, V and VIII). Representatives of 110 each neutralizing cluster were selected for evaluation against the Spike mutant pseudotypes.

\section{Impact of SARS-CoV Spike substitutions on SARS-CoV-2 mAb neutralization}

112 Initial screening assays of the twelve infectious viral pseudotype mutants showed no effect 113 on neutralization by any of the mAbs against three mutants with changes at amino acid 114 positions $\mathrm{RFA}_{346-8} \mathrm{KFP}, \mathrm{S}_{459} \mathrm{G}$, and $\mathrm{ST}_{477-8} \mathrm{GK}$ (FigS1). In contrast, the remaining nine viral 115 pseudotype mutants diminished neutralization for at least one mAb (Fig1) as described below:

$116 \mathbf{P}_{\mathbf{3 8 4}} \mathbf{A}$

117 The $\mathrm{P}_{384} \mathrm{~A}$ substitution resulted in complete loss of neutralization by COVA1-16 (Fig1A), a 118 cluster III RBD-specific mAb that allosterically competes with ACE2 rather than directly 119 blocking the binding site (Liu et al., 2020). Of note, this mutation has been described and 120 structurally characterized elsewhere (Wu et al., 2020), revealing that this proline to alanine 121 change results in a relatively small alteration in protein structure that can enable SARS-CoV $122 \mathrm{mAbs}$ to neutralize SARS-CoV-2 $\mathrm{P}_{384} \mathrm{~A}$. However, $\mathrm{P}_{384} \mathrm{~A}$ does not weaken neutralization by any 123 other mAbs, including another mAb in the cluster III competition group.

\section{$124 \mathbf{K}_{\mathbf{4 1 7}} \mathbf{V}$}

125 The $\mathrm{K}_{417} \mathrm{~V}$ mutation results in a pseudotyped virus that is less susceptible to COVA2-07 126 mediated neutralization, which is the other cluster III RBD-specific mAb screened (Fig1A). 127 COVA2-07 belongs to the same sub-cluster as COVA2-04, the structure of which has been solved 128 and contact residues include numerous bonded and non-bonded contacts within the RBD and 129 ACE2-binding site (Wu et al., 2020). That this mutation should alter mAbs such as COVA2-07, 130 which competes directly with ACE2 for binding, is not unexpected as the lysine at position 417 131 forms a hydrogen bond with ACE2 (Lan et al., 2020) that is likely disrupted by this substitution.

132 We then evaluated an additional $m A b, C O V A 2-04$, from the same competitive binding cluster as 133 COVA2-07. This is because COVA2-04 is representative of class of SARS-CoV-2 neutralizing 134 antibodies that all use the VH3-53 gene and have been suggested to be an unusual "public" or 135 stereotyped antibody (Cao et al., 2020; Mor et al., 2020; Robbiani et al., 2020). COVA2-04 was 136 not able to neutralize the $\mathrm{K}_{417} \mathrm{~V}$ pseudotype (data not shown). Although, of note, this mutation 137 has no real impact on neutralization by any of the other mAbs tested. 
This multiple substitution, which is a substantial change between SARS-CoV-2 and SARS-

$140 \mathrm{CoV}$, results in a 3.7-fold drop in neutralization potency for COVA2-29, which is a cluster I RBD-

141 specific antibody (Fig1A, B). This is the largest effect of this mutation, as the neutralization

142 activity of the other mAbs is largely unaffected despite the alteration of three sequential amino

143 acids. This may be explained by the relatively minor differences in the amino acid side chains at

144 the mutated residues. Unlike some of the other mutants tested, such as $\mathrm{LF}_{455} \mathrm{YL}$ that introduces

145 a phenol, the substitutions made here may not disrupt protein structure or antibody binding to

146 any great extent. Notably, binding analysis has suggested mutations in this loop can reduce RBD

147 binding by Spike-specific sera (Greaney et al., 2021).

\section{$148 \quad \mathbf{L}_{452} \mathbf{K}$}

149 This mutation is situated directly within the receptor binding motif (RBM) of the RBD. It 150 renders pseudotyped virus resistant to neutralization by the cluster I mAb COVA2-29, but does

151 not affect the other cluster I mAb COVA1-18 or any other mAb tested.

\section{$152 \quad \mathbf{L F}_{455} \mathbf{Y L}$}

153 This double substitution reduces neutralization by RBD-specific mAbs from different clusters, 154 specifically, the cluster I mAb COVA2-29, cluster III mAb COVA2-07 and cluster VI mAb COVA115512 (Fig1A, B). For COVA1-12 all neutralization activity is abolished, while COVA2-07 activity is 156 just below the level required to calculate an $\mathrm{IC}_{50}$ value.

\section{$157 \quad$ TEI $_{470-2} \mathbf{N V P}$}

158 This triple mutation is located in a loop within the RBM where other substitutions have been 159 reported to abolish ACE2 binding (Xu et al., 2021; Yi et al., 2020). This mutation prevents 160 neutralization by COVA2-29 (cluster I), COVA2-07 (cluster III) and COVA2-02 (cluster VII). It 161 also reduces the activity of the most potent mAb COVA1-18 (cluster I) by 3-fold, whereas this $162 \mathrm{mAb}$ is only minimally affected by other mutations. Moreover, $\mathrm{TEI}_{470-2} \mathrm{NVP}$ lowers the potency 163 of the structurally unmapped non-RBD cluster XI mAb, COVA1-21, to the limit of detection. 164 Thus, this mutation negatively impacts the most mAbs, including representatives from four 165 separate epitope clusters.

\section{S $_{494} \mathrm{D}$}

167 This single substitution towards the end of the RBM destroys neutralization activity by both 168 COVA2-29 (cluster I) and COVA1-12 (cluster VI) but does not have a major impact on the other 169 cluster I mAb tested or those from other epitope clusters. In summary, different mAbs can lose their neutralization activity when confronted with 171 different Spike mutations and the effects are not strictly delineated by binding clusters, such 
172

173

174

175

176

177

178

179

180

181

182

183

184

185

186

187

188

189

190

191

192

193

194

195

196

197

198

199

200

201

202

203

204

205

206

that mAbs within the same competition cluster are frequently differentially affected. The triple substitution $\mathrm{TEI}_{470-2 \mathrm{NVP}}$ has the most detrimental effects on different antibodies and impacts mAbs from nearly all binding clusters. $\mathrm{LF}_{455} \mathrm{YL}$ also negatively affects mAbs across different binding clusters. Notably, COVA2-29 is the mAb that is most frequently negatively affected by different mutations, with substantial loss of some neutralizing activity against four mutations (L452 K, LF 455 YL, TEI $470-2 \mathrm{NVP}, \mathrm{S}_{494} \mathrm{D}$ ), which are all within the RBM of the RBD (Fig1A). COVA2-29 is also known to compete directly with ACE2 for binding to Spike, which may explain why it is sensitive to so many different mutations within the RBM.

\section{Impact of Spike mutations on serum neutralization}

Following the identification of Spike mutations that can limit or abrogate neutralizing activity of mAbs (Fig2A), the next step was to assess the impact of these mutations on serum neutralization. Samples were tested following two different infection scenarios. Firstly, from a previously characterized cohort of seropositive healthcare workers who experienced mild or asymptomatic SARS-CoV-2 infection (Houlihan et al., 2020). Secondly, sera were obtained from a cohort of hospitalized patients who experienced severe disease. Eighteen samples were chosen from both cohorts for screening purposes to obtain representatives with intermediate $(1: 50-100)$, strong (1:100-1000) and potent (>1:1000) neutralizing $\mathrm{ID}_{50}$ values. The median serum $\mathrm{ID}_{50}$ for hospitalized patients selected was 1:1275, and that for selected mild/asymptomatic cases was 1:1045. Strikingly, serum samples from both cohorts are less impacted by Spike mutations than individual mAbs in terms of fold decrease in neutralization potency (Fig2B and C). Only one of thirty-six serum samples lost all neutralizing activity (Fig $\mathrm{S} 2$ ), in contrast to the five mAbs from five different epitope clusters where neutralization was completely abrogated by a single Spike mutation (Fig 1B). Moreover, fold-decrease in neutralization potency was more modest for sera than mAbs, with an average 2-fold decrease across all sera for the most disadvantageous mutation $\mathrm{TEI}_{470-2} \mathrm{NVP}$ as compared to a more than 100-fold decrease observed for several of the mAbs (Fig2B and C). Interestingly, none of the 36 serum samples lost $>5$-fold potency against the other triple substitution, $\mathrm{KVG}_{444-6} \mathrm{TST}$ which contrasts with recent data showing a single mutation at $\mathrm{G}_{446}$ caused a major loss of neutralization in one sample (Greaney et al., 2021). Importantly, there was notable difference between the resilience of serum samples from severely ill hospitalised individuals and those who had experienced mild/asymptomatic infection. Only two serum sample from a hospitalised individual lost more than 3-fold potency against any individual mutant (Fig2C, FigS2). Whereas approximately half (10 of 18 ) the mild/asymptomatic serum samples showed a three-fold drop in potency against at least one Spike mutant (Fig2C, Fig S2).

\section{Greater levels of Spike-reactive antibodies in sera after severe illness}


The differences in resilience to Spike mutations seen in the neutralizing sera from these two infection scenarios is plausibly due to greater polyclonality arising from greater antigenic stimulation during severe illness. To assess the serological profiles of these two cohorts, we

210 compared the $50 \%$ inhibitory dilution ( ID $_{50}$ ) values across 192 samples and measured the 211 binding titers by semi-quantitative ELISA for 199 samples as previously described (Ng et al., 212 2020; O'Nions et al., 2020). There is a significantly higher median IgG binding titer of 46.5 $213 \mu \mathrm{g} / \mathrm{ml}$ following severe illness versus $3.9 \mu \mathrm{g} / \mathrm{ml}$ following mild/asymptomatic disease (Fig3A, 214 FigS3). Similarly, there is a significantly higher median $\mathrm{ID}_{50}$ value in the hospitalized patient 215 cohort as compared to the mild/asymptomatic group (Fig3B). This shows, as has been 216 observed previously (Seow et al., 2020), that severely ill patients have higher binding and 217 neutralization titers than asymptomatic/mild cases and that higher binding titers correlate 218 with greater neutralization. However, when considering how the IgG binding titer from each 219 individual relates to their neutralization titer it became clear that there was a discrepancy 220 (Fig3C and D). Most hospitalized patients required a binding titer of greater than $10 \mu \mathrm{g} / \mathrm{ml}$ to 221 achieve strong neutralization $\left(\mathrm{ID}_{50}>100\right)$. Moreover, mild infections could lead to potent neutralization ( ID $_{50}>1000$ ) at binding titers of less than $10 \mu \mathrm{g} / \mathrm{ml}$ (Fig3D) whereas this was observed for only two individuals following severe illness (Fig3C). In fact, the amount of specific IgG present at the serum ID $_{50}$ is significantly higher in severe illness compared to mild disease (Fig 3E). This suggests that a higher proportion of antibodies are non-neutralizing following severe illness. However, the total level of specific antibodies is so high that the number of neutralizing antibodies following severe illness may be greater and explain the relative resilience of severe sera to Spike mutations.

\section{Impact of Spike variants on $\mathbf{m A b}$ and serum neutralization}

Investigating the ability of post-SARS-CoV-2-infection mAbs and serum to cope with mutations in Spike engineered based on differences with SARS-CoV was a rational first

232 approach to study escape, because these mutations were likely to form viable Spike proteins. 233 Indeed, some of the positions at which amino acids were mutated, as described above, have 234 now been observed to be a site of variance in SARS-CoV-2 across the human population (Q. Li et 235 al., 2020; Liu et al., 2020; Starr et al., 2020). However, additional viral variants, not linked to 236 points of variance between these two closely related viruses, have started to emerge on a 237 significant scale (Q. Li et al., 2020; Weisblum et al., 2020). Firstly, the $D_{614} G$ mutation, observed 238 in western Europe in February 2020 and now dominant across the globe (Korber et al., 2020). 239 It has already been described that $\mathrm{D}_{614} \mathrm{G}$ has higher infectivity and greater viral replication 240 (Korber et al., 2020; Q. Li et al., 2020; Plante et al., 2020) and somewhat increases the ability of 241 serum and mAbs to neutralize SARS-CoV-2 (Korber et al., 2020; Plante et al., 2020; Weissman et 242 al., 2020). More recently, a new variant of SARS-CoV-2 (B.1.1.7) has emerged in England and 
243 been associated with a rapid rise in case numbers (S. Kemp et al., 2020; Rambaut et al., 2020)

244 and high viral loads (Kidd et al., 2020). B.1.1.7 encodes 8 sites of change in Spike relative to the

245 original Wuhan strain. Of these, the most likely candidates to alter neutralization sensitivity are

246 the deletion in the NTD $\left(\Delta \mathrm{H}_{69} / \mathrm{V}_{70}\right)$ and the $\mathrm{N}_{501} \mathrm{Y}$ substitution in the RBM (S. Kemp et al., 2020;

247 Rambaut et al., 2020). Therefore, these changes were introduced in the Wuhan strain Spike in

248 the presence of $D_{614} G$ to produce pseudotyped viruses for neutralization sensitivity analysis.

249 Firstly, $\Delta \mathrm{H}_{69} / \mathrm{V}_{70}$ did not negatively impact the neutralization potency for most of the mAbs

250 tested, including cluster IX mAb COVA2-17 (Fig4A), which has recently been found to bind the

251 NTD (Rosa et al., 2021). The exception was the structurally unmapped COVA1-21 (cluster XI),

252 which was previously reported to lose partial potency against this deletion in the context of

253 other mutations that arose in an immuno-compromised patient (S. A. Kemp et al., 2020).

254 Similarly, the serum neutralization activity did not decrease by more than 3 -fold relative to the

$255 \mathrm{D}_{614} \mathrm{G}$ Spike for any individual sample (Fig4B). In contrast, the introduction of the $\mathrm{N}_{501} \mathrm{Y}$

256 substitution (observed in both B.1.1.7, 501Y.V2 and P.1) lowered the neutralization potency of

257 one mAb, COVA1-12, to the limit of the assay, with a fold decrease in $\mathrm{IC}_{50}$ of $>40$ (Fig4A). This

258 cluster VI RBD-specific mAb was also negatively impacted by proximal mutations at $\mathrm{LF}_{455} \mathrm{YL}$

259 and $\mathrm{S}_{494} \mathrm{D}$. Moreover, a 5-fold decrease in potency was observed against the $\mathrm{N}_{501} \mathrm{Y}$ pseudotype

260 for the cluster IX mAb COVA2-17, which also loses efficacy against TEI ${ }_{470-2}$ NVP. However, as

261 seen for the other mutations that abrogate mAb function, the $\mathrm{N}_{501} \mathrm{Y}$ change had less of an effect

262 on the sera obtained after both severe and mild infection, with all individual serum samples

263 remaining within three-fold of their original ID $_{50}$ value (Fig4B, FigS4).

\section{Impact of B.1.1.7 Spike on mAb and serum neutralization.}

265 Finally, a B.1.1.7 Spike pseudotyping plasmid was synthesized to incorporate the mutations 266 observed in this new variant in combination, and then evaluate neutralization sensitivity of 267 mAbs and sera. This showed that, as per individual mutants, B.1.1.7 can lessen the potency of 268 some mAbs, although unlike other mutations described above, it does not remove all activity 269 for any individual $\mathrm{mAb}$ and in total three mAbs were affected, COVA2-17, COVA1-12 and 270 COVA1-21 (Fig4A). These mAbs belong to distinct clusters and so do not compete for binding to 271 the same epitope. Firstly, the cluster IX mAb COVA2-17 showed an approximate 5-fold drop in 272 potency against both the $\mathrm{N}_{501} \mathrm{Y}$ single mutant and the B.1.1.7 pseudotype, implying this loss of 273 potency is primarily $\mathrm{N}_{501} \mathrm{Y}$ driven. In contrast, the decrease in potency noted with the single $274 \mathrm{~N}_{501} \mathrm{Y}$ change for the RBD-specific mAb COVA1-12 was lessened in the context of B.1.1.7, with a 275 11-fold rather than a >40-fold drop in neutralization. Furthermore, one mAb, COVA1-21 276 experienced a substantial drop in potency against B.1.1.7 compared to the single mutants or the $277 \mathrm{D}_{614} \mathrm{G}$ spike. This cluster XI mAb, which does not bind to RBD or S1 subunits, showed more than 278100 -fold reduction in potency. This effect is likely in part mediated by the $\Delta \mathrm{H}_{69} / \mathrm{V}_{70}$ deletion 
that also reduced the potency of COVA1-21 when evaluated as a single mutant. The B.1.1.7

280 Spike was then tested against the 36 serum samples from the two cohorts (Fig4B). The

281 maximum fold-decrease in potency for the serum samples from mild illness was 8.2 but the

282 majority of samples showed less than a 3-fold change. Similarly, the maximum decrease seen

283 for samples from hospitalized patients was a 9.1-fold change, but most of the samples showed

284 minimal change in the potency of their neutralization. Overall, three samples from each cohort

285 (8\%) showed a 5-10-fold reduction, but as they were potently neutralizing sera, the reduced

286 ID $_{50}$ values were still $>1: 100$. Similarly, another four samples from both cohorts $(11 \%)$ showed

287 a 3-5-fold reduction in neutralization when tested against the B.1.1.7 Spike pseudotype. Again,

288 the reduced $\mathrm{ID}_{50}$ values were still potent (on average $>1: 500$ ) with only two samples having an

$289 \quad \mathrm{ID}_{50}$ of $<1: 200$ (Fig S4)

\section{Discussion}

291 This study demonstrates that Spike mutations can diminish or abolish neutralizing activity by

292 individual mAbs but that serum neutralization is less strongly affected. Notably, only one

293 engineered mutation, and none of the observed Spike mutants or the B.1.1.7 variant, resulted in

294 a complete escape from neutralizing activity and this was only seen for one out of thirty-six

295 serum samples. The Spike mutants evaluated comprise seven substitutions designed to mimic

296 possible escape changes based on homology with SARS-CoV, two observed high-frequency

297 mutations and the B.1.1.7 Spike variant. The most likely explanation for the greater effect on

298 mAbs as compared to sera is the inherent polyclonality underlying serum neutralization. This

299 concept is supported by the observation that single Spike mutations can weaken neutralization

300 for a particular mAb but not for other mAbs from within the same binding cluster. This

301 highlights that different antibodies use distinct molecular contacts within shared epitopes, such

302 that a single mutation may not be detrimental to all antibodies within the same binding cluster.

303 Thus, because polyclonal sera contain multiple antibodies that target the major neutralizing

304 sites in subtly different ways, it is less sensitive to Spike mutations.

305 The Spike mutations studied here were designed to identify potential escape variants by 306 mimicking in part the natural variation observed between SARS-CoV and SARS-CoV-2, and are 307 focused mainly on the RBD as the major site of neutralizing antibody activity. Therefore, it was 308 unsurprising that many of the RBD-specific mAbs evaluated here lost neutralization activity 309 against one or more of these mutations. These included substantial multi-residue substitutions 310 not yet seen in the SARS-CoV-2 but also single point mutations at positions that have been 311 observed to mutate in the real world (S. Kemp et al., 2020; Korber et al., 2020; Rambaut et al., 312 2020; Tegally et al., 2020). These include, $\mathrm{K}_{417} \mathrm{~V}$ that had a negative effect on $\mathrm{mAb}$ 313 neutralization and another mutation at this position, $\mathrm{K}_{417} \mathrm{~N}$, has been observed in the emerging 
314 South African variant (Tegally et al., 2020). Importantly, the mAb (COVA2-07) that loses $>40$ -

315 fold neutralization activity against the $\mathrm{K}_{417} \mathrm{~V}$ pseudotyped virus belongs to the same cluster as 316 COVA2-04 that loses all neutralization activity against the $\mathrm{K}_{417} \mathrm{~V}$ mutant. COVA2-04 belongs to 317 the VH3-53 "public" BCR against SARS-CoV-2 identified from multiple human infections. Thus, 318 COVA2-04-like antibodies are thought to be widespread among the seropositive population and 319 so the $\mathrm{K}_{417} \mathrm{~V}$ would be predicted to have an impact on sera from many individuals. Interestingly, 320 serum samples from mild infection showed very little change in neutralization potency against 321 this mutation. However, the strongest effect on serum samples from mild infection was 322 mediated by the $\mathrm{TEI}_{470-2} \mathrm{NVP}$ substitution, which is part of what has been termed the RBD 323 binding ridge, and other mutations in this region can decrease serum neutralization (Greaney 324 et al., 2021). As such, any mutation in this zone should be closely monitored in viral populations 325 due to the potential for escape. However, it is encouraging that the effect of these mutations on sera was much less pronounced and that individual mAbs within the same clusters were not universally inactivated by any given mutation. Notably, the mutations that most substantially decrease serum neutralization are those that negatively impact mAb activity against the widest range of clusters (I, III, XI, IX and VI) suggesting that mAb screening is a useful proxy for potential serum effects if a range of antibody clones are used. However, the capacity to predict the in vivo impact of a drop in neutralization potency requires correlation of in vitro serum neutralization $\mathrm{ID}_{50}$ values with protection, which thus far has only been achieved in animal models where, encouragingly, an ID 50 value of 1:50 was found to be protective (McMahan et al., 334 2020).

A caveat to the first part of this study is that only RBD substitutions were considered. Further studies to assess potential mutations before they arise should include those in NTD given the emerging importance of NTD as a site for neutralizing antibodies (Andreano et al., 2020; Rosa et al., 2021). However, it should be noted that one RBD change, $\mathrm{TEI}_{470-2} \mathrm{NVP}$, resulted in a 24-fold drop in potency for COVA1-21, which does not bind RBD and remains structurally unmapped (Brouwer et al., 2020). Regardless, that sera likely containing a mixture of RBD- and NTD-specificities are more resilient than individual mAbs in the face of Spike mutations within the RBD is not surprising. This further highlights the importance of a broad

343 polyclonal serum response to maintain neutralizing activity in the event of novel Spike 344 mutations emerging, and the need to consider more than RBD binding in serological 345 evaluations.

346 To understand if the conclusions from studying the impact of the SARS-CoV-2/SARS-CoV 347 substitutions on neutralization parallel those of real-world Spike mutations, we examined the 348 responses to the newly emerged B.1.1.7 variant (S. Kemp et al., 2020; Rambaut et al., 2020). 
349 This revealed that the first NTD deletion observed, $\Delta \mathrm{H}_{69} / \mathrm{V}_{70}$, did not alter RBD-specific mAbs or 350 any sera. Although as previously described (S. A. Kemp et al., 2020) it did result in a drop in 351 potency for non-RBD mAb COVA1-21. The RBD mutation $\mathrm{N}_{501} \mathrm{Y}$, shared between B.1.1.7, 352 501Y.V2 and P.1, did remove almost all neutralizing activity for one mAb but, in a similar 353 pattern to other substitutions, this did not translate to any large effect on serum potency. Of 354 note, we have not studied changes at position 484 that have been observed in 501Y.V2 and P.1 and reported to reduce neutralization in serum samples (Greaney et al., 2021) and during in vitro escape (Andreano et al., 2020). Further studies of mutations at position 484 and new emerging mutations will be needed. This would best be facilitated by large, curated panels of mAbs and pools of sera from individuals with different infection/vaccination trajectories.

Theoretically it is likely that combinations of mutations have more potential to lead to loss of serum activity than individual single amino acid changes by destroying multiple parts of key epitopes. This has partially been observed in terms of the new B.1.1.7 Spike pseudotype analyzed here. Only one mAb was more dramatically affected by the full set of B.1.1.7 mutations. However, the small number of serum samples with reduced neutralization relative

364 to the $\mathrm{D}_{614} \mathrm{G}$ virus, were more strongly affected by B.1.1.7 than either the $\Delta \mathrm{H}_{69} / \mathrm{V}_{70}$ or $\mathrm{N}_{501} \mathrm{Y}$ mutations individually (Fig 4B). This reduced neutralization was seen in 11\% (3-5-fold) and 8\% (5-10-fold) when tested against the B.1.1.7 Spike pseudotype. However, all of the affected samples were still able to neutralize B.1.1.7, and the average reduced serum ID $_{50}$ value was 1:522. This is ten-times higher than the reported serum $\mathrm{ID}_{50}$ correlate of protection in animal studies and suggests these responses would likely still be effective against infection with B.1.1.7.

The differences in the data observed with B.1.1.7 and the two individual mutations $\Delta \mathrm{H}_{69} / \mathrm{V}_{70}$ or $\mathrm{N}_{501} \mathrm{Y}$ highlight the importance of testing emerging variants in the full form.

373 Moreover, this approach may be important as combinations of mutations could enable 374 individual antibody escape mutations that are disadvantageous for transmission to be 375 propagated. For example, a residue such as $\mathrm{S}_{494}$ is involved both in ACE2 binding (Xu et al., 376 2021) and mAb neutralization (Fig1). Therefore, a mutation at $S_{494}$ could decrease antibody 377 function but also decrease host receptor recognition and limit infectivity. However, the 378 detrimental effects of the mutation on infectivity could be compensated for if a $\mathrm{S}_{494}$ mutation 379 occurred in concert with a mutation that strengthened a different part of the viral entry 380 pathway as has been suggested(S. A. Kemp et al., 2020). This highlights the need for rapid 381 evaluation of variant strains upon emergence, potentially accelerated by computational modeling based on prior knowledge of the effects of individual changes. 
In conclusion, this study underlines both the potential for escape from neutralizing antibodies due to mutations in Spike and the relative resilience of serum responses compared to individual mAbs. This difference likely derives from the breadth inherent in polyclonal sera as compared to the precision interaction of a given $\mathrm{mAb}$. Our results suggest that the majority of vaccine responses should be effective against the B.1.1.7 variant as the sera evaluated were obtained after infection early in the pandemic when the commonly circulating virus was highly similar in sequence to the vaccines now being deployed. A reduction in potency was observed in a minority of samples tested against B.1.1.7, however, neutralization titers remained above 1:200 in almost all cases. Finally, it is probable that as SARS-CoV-2 seropositivity increases across the human population (due to both vaccination efforts and natural infection) there could eventually be selection for Spike mutations that result in substantial antigenic drift as seen for Influenza. If and when this will happen is unpredictable given the current scale of ongoing transmission worldwide. The data herein suggest evaluation of neutralizing mAbs from nonoverlapping binding clusters can highlight which Spike mutations will most impact sera neutralization. Greater knowledge of the molecular epitopes recognized by individual mAbs and their relative immunodominance within sera is needed urgently. This is because understanding of rules of engagement for SARS-CoV-2 neutralizing antibodies is a crucial component of preparedness for major antigenic changes and long-term management of coronaviruses globally. Our findings stress the importance of continuous monitoring of variants and in vitro assessment of their impact on neutralization. This is particularly relevant for the use of convalescent plasma and the development of therapeutic monoclonal antibodies as well as vaccine development and implementation.

\section{Acknowledgements}

406 The authors would like to thank James E Voss for the gift of HelaACE2 expressing cells, George 407 Kassiotis, Dan Frampton, Ann-Kathrin Reuschl and Joe Grove for helpful discussion and critical 408 feedback. We are indebted to the Biobank staff and study participants and their families at the 409 Royal Free Hospital, and the UCLH SAFER study recruitment team and study participants.

\section{Funding}

412 This study was funded by the UCL Coronavirus Response Fund made possible through 413 generous donations from UCL's supporters, alumni and friends (LEM) and also by the King's 414 Together Rapid COVID-19 Call award (KJD), the Huo Family Foundation (KJD). This research 415 was also funded by the Royal Free Charity. LEM is supported by a Medical Research Council 416 Career Development Award (MR/R008698/1). MJvG is a recipient of an AMC Fellowship, and 417 RWS is a recipient of a Vici grant from the Netherlands Organization for Scientific Research 418 (NWO). C.G. is supported by the MRC-KCL Doctoral Training Partnership in Biomedical Sciences 
419 (MR/N013700/1). This work was also supported by National Institutes of Health grant P01

420 AI110657, and by the Bill and Melinda Gates Foundation grant INV-002022 (RWS). The work in

421 laboratory of PC was supported by the Francis Crick Institute (FC001061), which receives its

422 core funding from Cancer Research UK, the UK Medical Research Council, and the Wellcome

423 Trust. The SAFER study was funded by MRC UKRI (grant MC_PC_19082) and supported by the

424 UCLH/UCL NIHR BRC.

\section{Author contributions}

426 KJD, LEM, LM, SAG, PT, NL and CR-S characterized monoclonal antibodies and sera; AR, CR, and

427 PC expressed and purified proteins; JH, CH, HS and EN assembled the panels of human sera

428 samples; MJvG, RWS, YA, JLS isolated and provided monoclonal antibodies; RG generated and

429 provided mutated Spike plasmids, MJvG, KJD, and LEM wrote the paper with contributions from

430 all authors. 


\section{Methods}

\section{Spike mutant generation}

433 QuikChange Lightening Site-Directed Mutagenesis kit was used to generate amino acid 434 substitutions in the SARS-CoV-2 Wuhan Spike expression vector (Seow et al., 2020) or the 435 D614G pCDNA Spike plasmid (S. A. Kemp et al., 2020) following the manufacturer's instructions 436 (Agilent Technologies, Inc., Santa Clara, CA). Spike B.1.1.7 was synthesised by Genewiz, Inc. and 437 cloned into the pCDNA expression vector using BamHI and EcoRI restriction sites.

\section{Neutralization assay}

439 HIV-1 particles pseudotyped with SARS-Cov-2 spike were produced in a T75 flask seeded the 440 day before with 3 million HEK293T cells in $10 \mathrm{ml}$ complete DMEM, supplemented with 10\% 441 FBS, $100 \mathrm{IU} / \mathrm{ml}$ penicillin and $100 \mu \mathrm{g} / \mathrm{ml}$ streptomycin. Cells were transfected using $60 \mu \mathrm{g}$ of 442 PEI-Max (Polysciences) with a mix of three plasmids: $9.1 \mu \mathrm{g}$ HIV-1 luciferase reporter vector 443 (Seow et al., 2020), $9.1 \mu \mathrm{g}$ HIV p8.91 packaging construct and $1.4 \mu \mathrm{g}$ WT SARS-CoV-2 spike 444 expression vector (Seow et al., 2020). Supernatants containing pseudotyped virions were 445 harvested $48 \mathrm{~h}$ post-transfection, filtered through a $0.45-\mu \mathrm{m}$ filter and stored at $-80^{\circ} \mathrm{C}$. 446 Neutralization assays were conducted by serial dilution of monoclonal IgG at the indicated 447 concentrations in DMEM (10\% FBS and 1\% penicillin-streptomycin) and incubated with 448 pseudotyped virus for $1 \mathrm{~h}$ at $37^{\circ} \mathrm{C}$ in 96-well plates. HeLa cells stably expressing ACE-2 449 (provided by J.E. Voss, Scripps Institute) were then added to the assay $(10,000$ cells per $100 \mu \mathrm{l}$ 450 per well). After 48-72 h luminescence was assessed as a proxy of infection by lysing cells with 451 the Bright-Glo luciferase kit (Promega), using a Glomax plate reader (Promega). Measurements 452 were performed in duplicate and used to calculate $50 \%$ inhibitory concentrations ( $\mathrm{IC}_{50}$ ) in 453 GraphPad Prism software.

\section{Semi-quantitative ELISA}

455 As described previously (O'Nions et al., 2020) nine columns of a half-well 96-well MaxiSorp 456 plate were coated with purified SARS-CoV-2 Spike S1 protein in PBS ( $3 \mu \mathrm{g} / \mathrm{ml}$ per well in $25 \mu \mathrm{L}$ ) 457 and the remaining three columns were coated with $25 \mu \mathrm{L}$ goat anti-human F(ab)'2 diluted 458 1:1000 in PBS to generate the internal standard curve. After incubation at $4^{\circ} \mathrm{C}$ overnight, the 459 ELISA plate was blocked for 1 hour in assay buffer (PBS, 5\% milk, 0.05\% Tween 20). Sera was 460 diluted in assay buffer at dilutions from 1:50 to 1:5000 and $25 \mu \mathrm{L}$ added to the ELISA plate. 461 Serial dilutions of known concentrations of IgG standards were applied to the three standard 462 curve columns in place of sera. The ELISA plate was then incubated for 2 hours at room 463 temperature and then washed 4 times with PBS-T (PBS, 05\% Tween 20). Alkaline phosphatase-

464 conjugated goat anti-human IgG at a 1:1000 dilution was then added to each well and incubated 465 for 1 hour. Following this, plates were washed 6 times with PBS-T and $25 \mu \mathrm{L}$ of colorimetric 
466 alkaline phosphatase substrate added. Absorbance was measured at $405 \mathrm{~nm}$. Antigen-specific

467 IgG concentrations in serum were then calculated based on interpolation from the IgG standard

468 results using a four-parameter logistic (4PL) regression curve fitting model.

469 Serum samples

470 SAFER study samples were collected as previously described (Houlihan et al., 2020). Data

471 derives from samples from 81 seropositive individuals during the first four months since

472 infection. The study protocol was approved by the NHS Health Research Authority (ref

473 20/SC/0147) on 26 March 2020. Ethical oversight was provided by the South- Central

474 Berkshire Research Ethics Committee. Serum samples from hospitalised patients were

475 obtained during their hospital stay and through the Tissue Access for Patient Benefit (TAPb)

476 scheme at The Royal Free Hospital (approved by UCL-Royal Free Hospital BioBank Ethical

477 Review Committee Reference number: NC2020.24 NRES EC number: 16/WA/0289). 
References

Andreano, E., Piccini, G., Licastro, D., Casalino, L., Johnson, N. V., Paciello, I., . . Rappuoli, R. (2020). SARS-CoV-2 escape <em>in vitro</em> from a highly neutralizing COVID-19 convalescent plasma. bioRxiv.

Brouwer, P. J. M., Caniels, T. G., van der Straten, K., Snitselaar, J. L., Aldon, Y., Bangaru, S., . . van Gils, M. J. (2020). Potent neutralizing antibodies from COVID-19 patients define multiple targets of vulnerability. Science, 369(6504), 643-650. doi:10.1126/science.abc5902

Burton, D. R., Poignard, P., Stanfield, R. L., \& Wilson, I. A. (2012). Broadly Neutralizing Antibodies Present New Prospects to Counter Highly Antigenically Diverse Viruses. Science, 337, 183-186.

Cao, Y., Su, B., Guo, X., Sun, W., Deng, Y., Bao, L., ... Xie, X. S. (2020). Potent Neutralizing Antibodies against SARS-CoV-2 Identified by High-Throughput Single-Cell Sequencing of Convalescent Patients' B Cells. Cell, 182(1), 73-84.e16. doi:10.1016/j.cell.2020.05.025

CDC. (2003). Update: Outbreak of severe acute respiratory syndrome--worldwide, 2003. MMWR Morb Mortal Wkly Rep, 52(12), 241-246, 248.

Cevik, M., Kuppalli, K., Kindrachuk, J., \& Peiris, M. (2020). Virology, transmission, and pathogenesis of SARS-CoV-2. BMJ, 371.

Faria, N. R., Claro, I. M., Candido, D., Moyses Franco, L. A., Andrade, P. S., Coletti, T. M., ... Sabino, E. C., on behalf of CADDE Genomic Network. (2021). Genomic characterisation of an emergent SARS-CoV-2 lineage in Manaus: preliminary findings.

Gralinski, L. E., \& Menachery, V. D. (2020). Return of the Coronavirus: 2019-nCoV. Viruses, 12(2), 135.

Greaney, A. J., Loes, A. N., Crawford, K. H. D., Starr, T. N., Malone, K. D., Chu, H. Y., \& Bloom, J. D. (2021). Comprehensive mapping of mutations to the SARS-CoV-2 receptor-binding domain that affect recognition by polyclonal human serum antibodies. bioRxiv.

Houlihan, C. F., Vora, N., Byrne, T., Lewer, D., Kelly, G., Heaney, J., ... Nastouli, E. (2020). Pandemic peak SARS-CoV-2 infection and seroconversion rates in London frontline health-care workers. Lancet, 396(10246), e6-e7. doi:10.1016/s0140-6736(20)31484-7

Kemp, S., Datir, R., Collier, D., Ferreira, I., Carabelli, A., Harvey, W., .. Gupta, R. (2020). Recurrent emergence and transmission of a SARS-CoV-2 Spike deletion $\Delta \mathrm{H} 69 / \Delta \mathrm{V} 70$. bioRxiv.

Kemp, S. A., Collier, D. A., Datir, R., Ferreira, I. A., Gayed, S., Jahun, A., ... Gupta, R. K. (2020). Neutralising antibodies drive Spike mediated SARS-CoV-2 evasion. medRxiv.

Kidd, M., Richter, A., Best, A., Mirza, J., Percival, B., Mayhew, M., ... McNally, A. (2020). S-variant SARS-CoV-2 is associated with significantly higher viral loads in samples tested by ThermoFisher TaqPath RT-QPCR. medRxiv.

Korber, B., Fischer, W. M., Gnanakaran, S., Yoon, H., Theiler, J., Abfalterer, W., ... Montefiori, D. C. (2020). Tracking Changes in SARS-CoV-2 Spike: Evidence that D614G Increases Infectivity of the COVID-19 Virus. Cell. doi:10.1016/j.cell.2020.06.043

Lan, J., Ge, J., Yu, J., Shan, S., Zhou, H., Fan, S., . . Wang, X. (2020). Structure of the SARS-CoV-2 spike receptor-binding domain bound to the ACE2 receptor. Nature, 581(7807), 215220. doi:10.1038/s41586-020-2180-5

Li, Q., Wu, J., Nie, J., Zhang, L., Hao, H., Liu, S., .. Wang, Y. (2020). The Impact of Mutations in SARS-CoV-2 Spike on Viral Infectivity and Antigenicity. Cell, 182(5), 1284-1294.e1289. doi:10.1016/j.cell.2020.07.012

Li, W., Moore, M. J., Vasilieva, N., Sui, J., Wong, S. K., Berne, M. A., . . Farzan, M. (2003). Angiotensin-converting enzyme 2 is a functional receptor for the SARS coronavirus. Nature, 426(6965), 450-454. doi:10.1038/nature02145

Lipsitch, M., Cohen, T., Cooper, B., Robins, J. M., Ma, S., James, L., ... Murray, M. (2003). Transmission Dynamics and Control of Severe Acute Respiratory Syndrome. Science, 300, 1966-1970. 
Liu, H., Wu, N. C., Yuan, M., Bangaru, S., Torres, J. L., Caniels, T. G., .. . Wilson, I. A. (2020). CrossNeutralization of a SARS-CoV-2 Antibody to a Functionally Conserved Site Is Mediated by Avidity. Immunity, 53(6), 1272-1280.e1275. doi:10.1016/j.immuni.2020.10.023

McMahan, K., Yu, J., Mercado, N. B., Loos, C., Tostanoski, L. H., Chandrashekar, A., . . Barouch, D. H. (2020). Correlates of protection against SARS-CoV-2 in rhesus macaques. Nature. doi:10.1038/s41586-020-03041-6

Mor, M., Werbner, M., Alter, J., Safra, M., Chomsky, E., Hada-Neeman, S., . . Freund, N. T. (2020). Multi-Clonal Live SARS-CoV-2 In Vitro Neutralization by Antibodies Isolated from Severe COVID-19 Convalescent Donors. bioRxiv.

Ng, K. W., Faulkner, N., Cornish, G. H., Rosa, A., Harvey, R., Hussain, S., .. Kassiotis, G. (2020). Preexisting and de novo humoral immunity to SARS-CoV-2 in humans. Science, 370(6522), 1339-1343. doi:10.1126/science.abe1107

O'Nions, J., Muir, L., Zheng, J., Rees-Spear, C., Rosa, A., Roustan, C., ... McCoy, L. E. (2020). SARSCoV-2 antibody responses in patients with acute leukaemia. Leukemia, 1-4. doi:10.1038/s41375-020-01103-2

Ortega, J. T., Serrano, M. L., Pujol, F. H., \& Rangel, H. R. (2020). Role of changes in SARS-CoV-2 spike protein in the interaction with the human ACE2 receptor: An in silico analysis. Excli j, 19, 410-417. doi:10.17179/excli2020-1167

Oude Munnink, B. B., Sikkema, R. S., Nieuwenhuijse, D. F., Molenaar, R. J., Munger, E., Molenkamp, R., ... Koopmans, M. P. G. (2020). Transmission of SARS-CoV-2 on mink farms between humans and mink and back to humans. Science.

Petersen, E., Koopmans, M., Go, U., Hamer, D. H., Petrosillo, N., Castelli, F., . . Simonsen, L. (2020). Comparing SARS-CoV-2 with SARS-CoV and influenza pandemics. The Lancet Infectious Diseases, 20(9), e238-e244. doi:10.1016/S1473-3099(20)30484-9

Plante, J. A., Liu, Y., Liu, J., Xia, H., Johnson, B. A., Lokugamage, K. G., . . Shi, P.-Y. (2020). Spike mutation D614G alters SARS-CoV-2 fitness. Nature. doi:10.1038/s41586-020-2895-3

Plotkin, S. A. (2008). Correlates of Vaccine-Induced Immunity. Clinical Infectious Diseases, 47(3), 401-409. doi:10.1086/589862

Polack, F. P., Thomas, S. J., Kitchin, N., Absalon, J., Gurtman, A., Lockhart, S., . . Gruber, W. C. (2020). Safety and Efficacy of the BNT162b2 mRNA Covid-19 Vaccine. New England Journal of Medicine. doi:10.1056/NEJMoa2034577

Rambaut, A., Loman, N., Pybus, O., Barclay, W., Barrett, J., Carabelli, A., ... Volz, E. (2020). Preliminary genomic characterisation of an emergent SARS-CoV-2 lineage in the UK defined by a novel set of spike mutations.

Robbiani, D. F., Gaebler, C., Muecksch, F., Lorenzi, J. C. C., Wang, Z., Cho, A., ... Nussenzweig, M. C. (2020). Convergent antibody responses to SARS-CoV-2 in convalescent individuals. Nature, 584(7821), 437-442. doi:10.1038/s41586-020-2456-9

Rockx, B., Donaldson, E., Frieman, M., Sheahan, T., Corti, D., Lanzavecchia, A., \& Baric, R. S. (2010). Escape from human monoclonal antibody neutralization affects in vitro and in vivo fitness of severe acute respiratory syndrome coronavirus. J Infect Dis, 201(6), 946955. doi:10.1086/651022

Rosa, A., Pye, V. E., Graham, C., Muir, L., Seow, J., Ng, K. W., ... Cherepanov, P. (2021). SARS-CoV2 recruits a haem metabolite to evade antibody immunity. In preparation.

Seow, J., Graham, C., Merrick, B., Acors, S., Pickering, S., Steel, K. J. A., ... Doores, K. J. (2020). Longitudinal observation and decline of neutralizing antibody responses in the three months following SARS-CoV-2 infection in humans. Nat Microbiol, 5(12), 1598-1607. doi:10.1038/s41564-020-00813-8

Starr, T. N., Greaney, A. J., Hilton, S. K., Ellis, D., Crawford, K. H. D., Dingens, A. S., . . Bloom, J. D. (2020). Deep Mutational Scanning of SARS-CoV-2 Receptor Binding Domain Reveals Constraints on Folding and ACE2 Binding. Cell, 182(5), 1295-1310.e1220. doi:10.1016/j.cell.2020.08.012

Tegally, H., Wilkinson, E., Giovanetti, M., Iranzadeh, A., Fonseca, V., Giandhari, J., . . d de Oliveira, T. (2020). Emergence and rapid spread of a new severe acute respiratory syndrome- 
related coronavirus 2 (SARS-CoV-2) lineage with multiple spike mutations in South Africa. medRxiv.

Weisblum, Y., Schmidt, F., Zhang, F., DaSilva, J., Poston, D., Lorenzi, J. C., ... Bieniasz, P. D. (2020). Escape from neutralizing antibodies by SARS-CoV-2 spike protein variants. Elife, 9. doi:10.7554/eLife.61312

Weissman, D., Alameh, M.-G., de Silva, T., Collini, P., Hornsby, H., Brown, R., . . Montefiori, D. C. (2020). D614G Spike Mutation Increases SARS CoV-2 Susceptibility to Neutralization. Cell Host \& Microbe. doi:https://doi.org/10.1016/j.chom.2020.11.012

Welkers, M. R. A., Han, A. X., Reusken, C. B. E. M., \& Eggink, D. (2020). Possible host-adaptation of SARS-CoV-2 due to improved ACE2 receptor binding in mink. Virus evolution. doi:10.1093/ve/veaa094

Wu, N. C., Yuan, M., Bangaru, S., Huang, D., Zhu, X., Lee, C. D., .. W Wilson, I. A. (2020). A natural mutation between SARS-CoV-2 and SARS-CoV determines neutralization by a crossreactive antibody. PLoS Pathog, 16(12), e1009089. doi:10.1371/journal.ppat.1009089

Xie, X., Zou, J., Fontes-Garfias, C. R., Xia, H., Swanson, K. A., Cutler, M., ... Shi, P.-Y. (2021). Neutralization of N501Y mutant SARS-CoV-2 by BNT162 b2 vaccine-elicited sera. bioRxiv.

Xu, C., Wang, Y., Liu, C., Zhang, C., Han, W., Hong, X., . . Cong, Y. (2021). Conformational dynamics of SARS-CoV-2 trimeric spike glycoprotein in complex with receptor ACE2 revealed by cryo-EM. Science Advances, 7.

Yi, C., Sun, X., Ye, J., Ding, L., Liu, M., Yang, Z., ... Sun, B. (2020). Key residues of the receptor binding motif in the spike protein of SARS-CoV-2 that interact with ACE2 and neutralizing antibodies. Cellular \& Molecular Immunology, 17(6), 621-630. doi:10.1038/s41423-020-0458-z

Zost, S. J., Wu, N. C., Hensley, S. E., \& Wilson, I. A. (2019). Immunodominance and Antigenic Variation of Influenza Virus Hemagglutinin: Implications for Design of Universal Vaccine Immunogens. The Journal of Infectious Diseases, 219(Supplement_1), S38-S45. 


\section{Figures}

613 Figure 1: Mutating amino acids in SARS-CoV-2 Spike to match SARS-CoV decreases mAb

614 neutralization. (A) Indicated mAbs were serially diluted in duplicate and incubated with each

615 mutant SARS-CoV-2 luciferase-encoding pseudotyped virus (as noted in the legend) prior to the

616 addition of HeLa cells expressing ACE-2. After two days, neutralization was measured as the

617 relative reduction in relative light units (RLU). Data are representative of three independent

618 repeats. The horizontal dotted line on each graph indicates 50\% neutralization. (B) 50\%

619 inhibitory concentration $\left(\mathrm{IC}_{50}\right)$ values were calculated for each mAb against the mutant SARS-

620 CoV-2 pseudotyped viruses indicated in the left hand column. $\mathrm{IC}_{50}$ values are color-coded as

621 follows: pale grey $>1 \mu \mathrm{g} / \mathrm{ml}$, light grey $0.1-1 \mu \mathrm{g} / \mathrm{ml}$, medium grey $0.1-0.01 \mu \mathrm{g} / \mathrm{ml}$ and dark grey

$622<0.01 \mu \mathrm{g} / \mathrm{ml}$. The previously established binding cluster for each $\mathrm{mAb}$ is indicated above each

623 column, and whether or not the mAb binds RBD is also indicated. 
bioRxiv preprint doi: https//doi.org/10.1101/2021.01.15.426849; this version posted January 19,2021 . The copyright holder for this preprint (which was not certified by peer review) is the author/funder, who has granted bioRxiv a license to display the preprint in perpetuity. It is made available under aCC-BY-ND 4.0 International license.

FIGURE 1

A
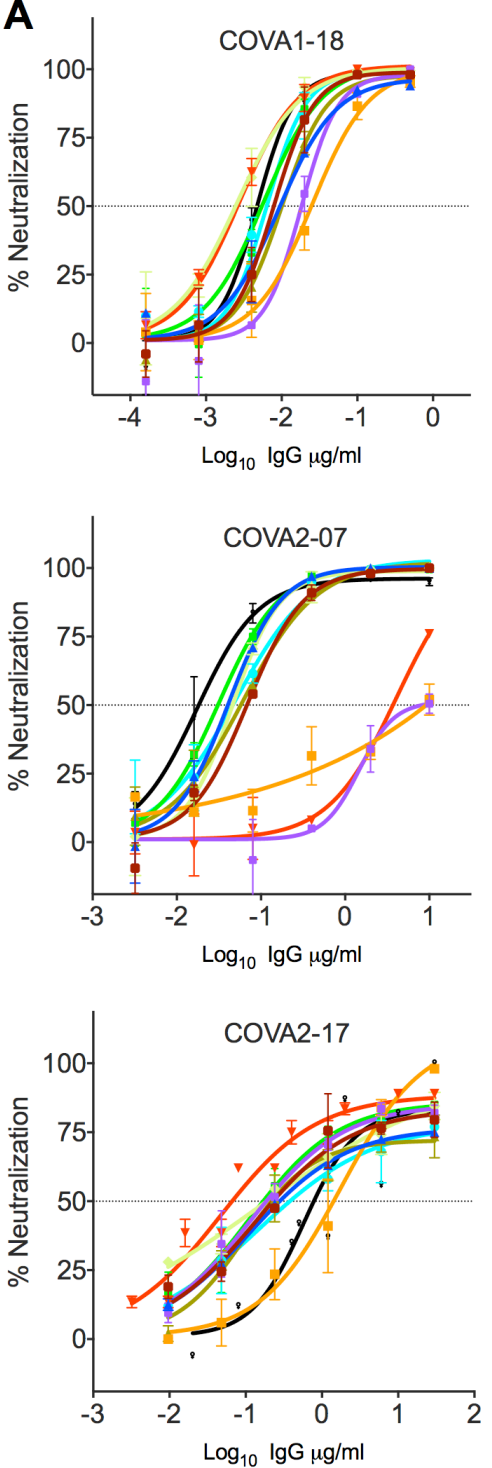

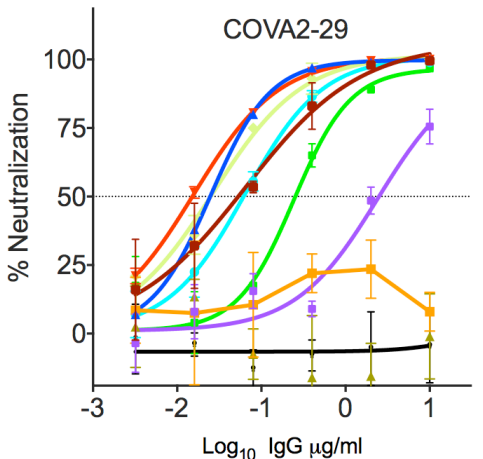

COVA1-12
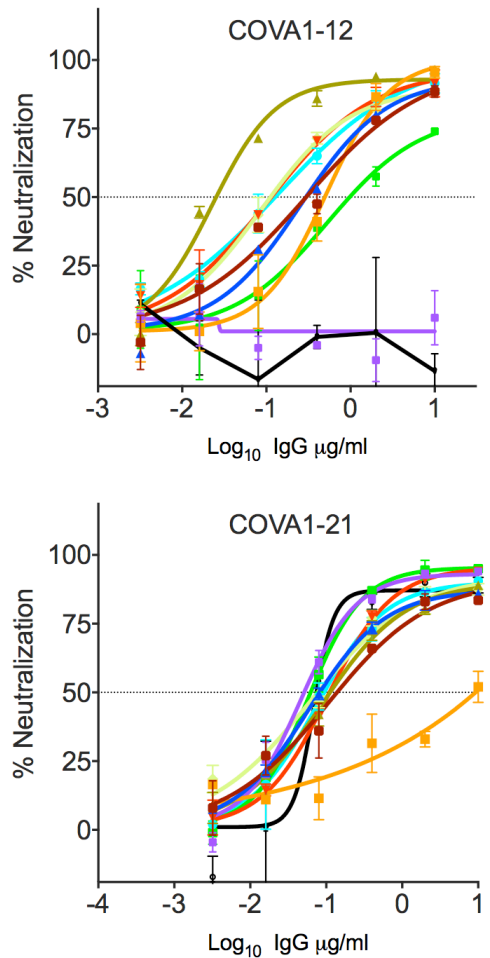

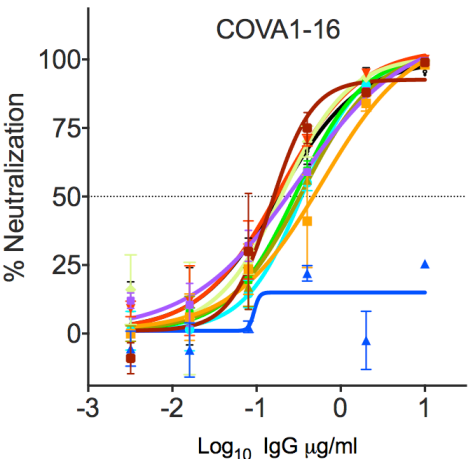

COVA2-02

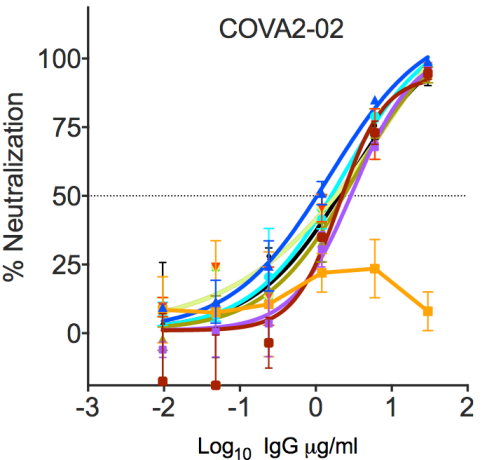

$$
\begin{aligned}
& -\mathrm{WT} \\
& =\mathrm{ES}_{324-5} \mathrm{GD} \\
& -\mathrm{S}_{373} \mathrm{~F} \\
& -\mathrm{P}_{384} \mathrm{~A} \\
& =\mathrm{K}_{417} \mathrm{~V} \\
& =\mathrm{L}_{452} \mathrm{~K} \\
& =\mathrm{LF}_{455} \mathrm{YL} \\
& =\mathrm{TEI}_{470-2} \mathrm{NVP} \\
& -\mathrm{KVG}_{444-6} \mathrm{TST} \\
& -\mathrm{S}_{494} \mathrm{D}
\end{aligned}
$$

B

RBD

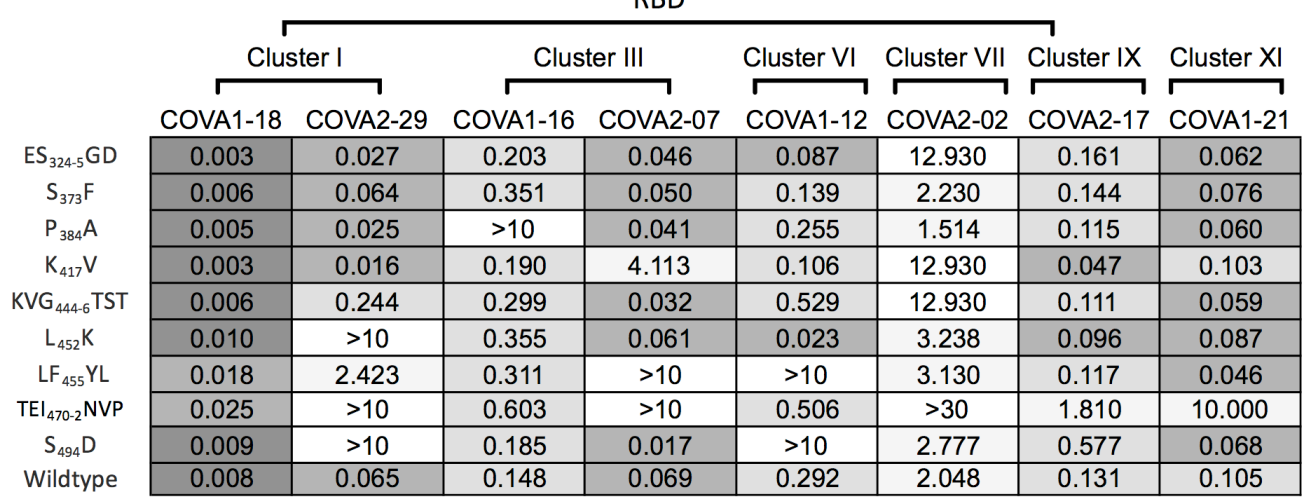


624 Figure 2: Neutralization by sera is less adversely affected by SARS-CoV amino acid 625 substitutions in SARS-CoV-2 Spike. (A) Representation of SARS CoV-2 Spike trimer (blue) in 626 complex with ACE-2 (pink) (PDB code 7DF4). Magnified image shows mutated amino acid side 627 chains at residues of interest. (B) $\mathrm{IC}_{50}$ values for each $\mathrm{mAb}$ against SARS-CoV-2 wildtype 628 pseudotyped virus were divided by the $\mathrm{IC}_{50}$ for each mutant pseudotyped virus against the 629 corresponding $\mathrm{mAb}$ to generate the fold decrease in neutralization on the Y-axis. The dotted 630 horizontal line indicates a 5-fold drop in neutralization potency. The competitive binding 631 clusters of each mAb that loses > 5-fold neutralization activity are labeled on the graph. (C)

632 Thirty six serum samples were serially titrated and incubated with the mutant SARS-CoV-2 633 luciferase-encoding pseudotyped viruses indicated in the legend prior to the addition of HeLa 634 cells expressing ACE-2. After two days, neutralization was measured as the relative reduction in 635 relative light units (RLU) and 50\% inhibitory dilution factors calculated using Graphpad Prism. 636 ID $_{50}$ values for each sera against SARS-CoV-2 wildtype pseudotyped virus were divided by the $637 \mathrm{ID}_{50}$ for each mutant pseudotyped virus against the corresponding sera to generate the fold 638 decrease in neutralization on the Y-axis. The dotted horizontal line indicates a 5-fold drop in 639 neutralization potency. The 18 serum samples from hospitalized patients are shown in the 640 upper graph labeled "severe illness" and the 18 serum samples from healthcare workers who 641 experience mild/asymptomatic COVID-19 are shown in the lower graph labeled "mild illness". 
A

B

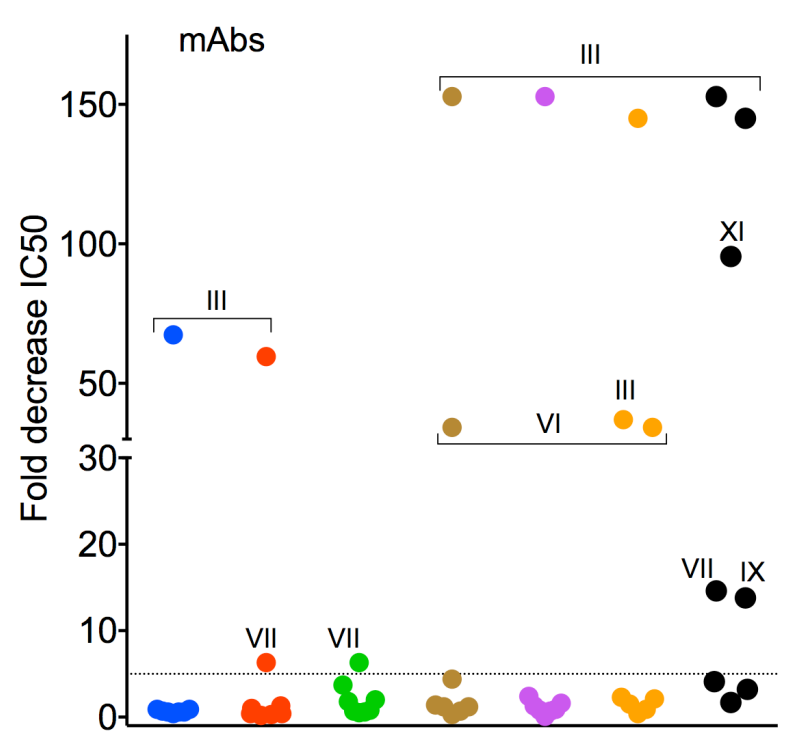

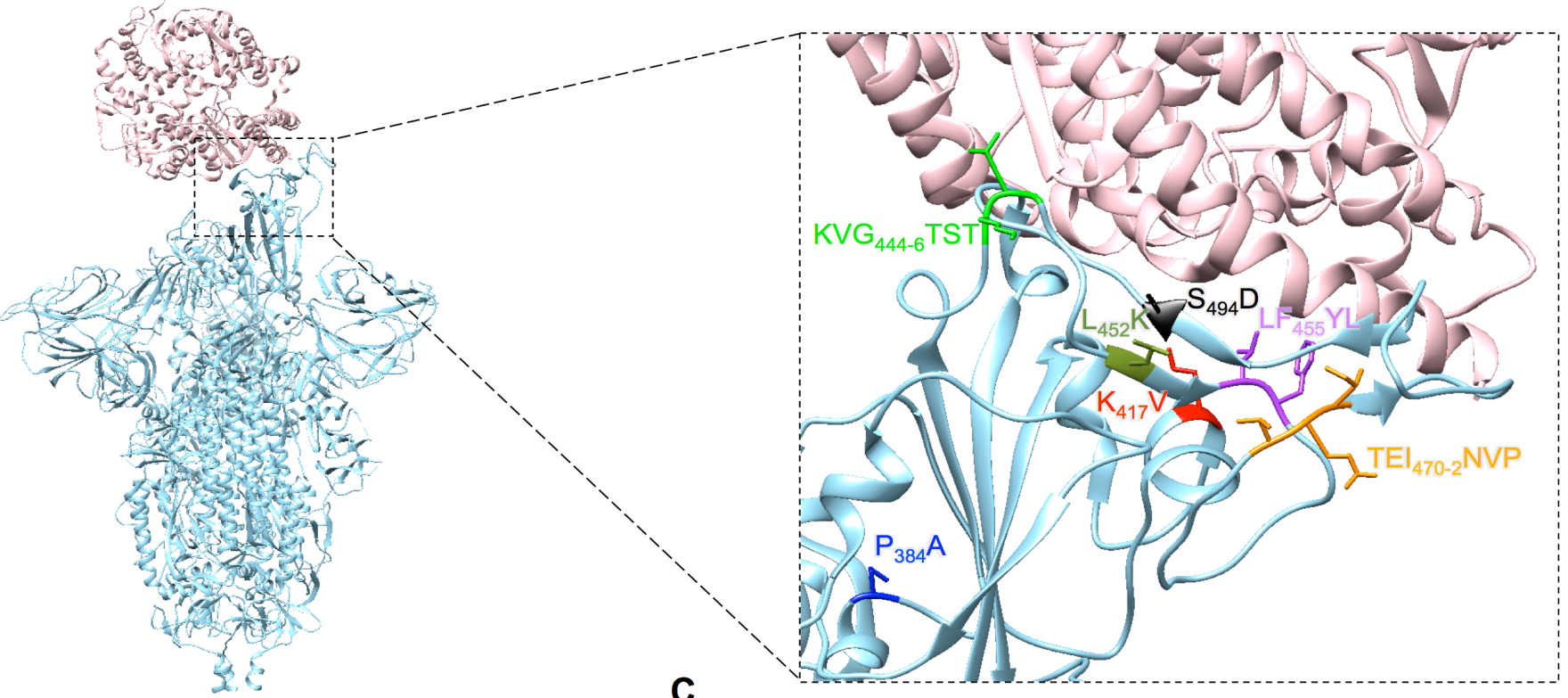

C

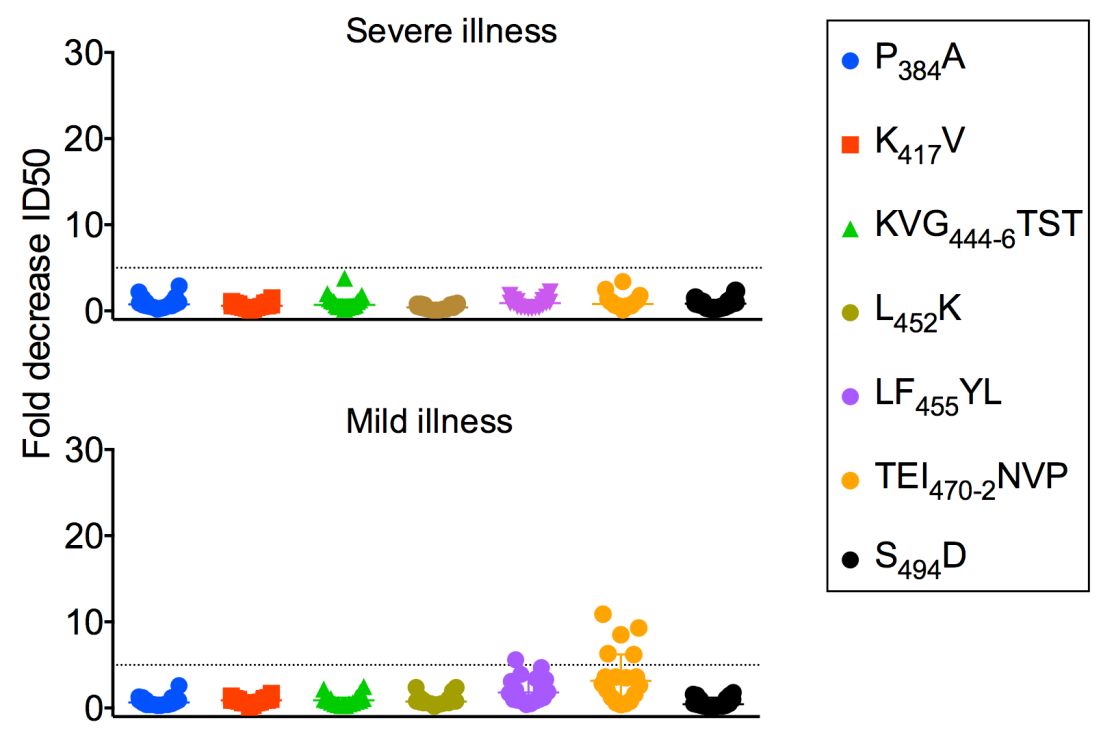


643 Figure 3: Serum responses following severe COVID-19 have greater polyclonality but less

644 efficient neutralization. (A) Spike S1 subunit semi-quantitative titers measured by ELISA (see

645 Methods) are shown on the Y-axis for 94 serum samples from hospitalized COVID-19 patients

646 and 105 serum samples from healthcare workers who experienced mild COVID-19 disease. (B)

647 Serum ID 50 values measured by pseudotyped neutralization assay (see Methods) are shown on

648 the X-axis for 93 serum samples from hospitalized COVID-19 patients and 99 serum samples

649 from healthcare workers who experienced mild COVID-19 disease. Note, some serum samples

650 from the original cohort that had binding titers gave no neutralization titer (6 from healthcare

651 workers, 1 from a hospitalized patient). (C) ID $_{50}$ values measured by pseudotyped

652 neutralization assay for serum samples from hospitalized COVID-19 patients plotted on the Y-

653 axis against the corresponding S1 IgG binding titer for each sample. Relative ranking of

654 neutralization titers is indicated on the graph. (D) Serum ID $_{50}$ values measured by pseudotyped

655 neutralization assay for the serum samples from healthcare workers who experienced mild

656 COVID-19 disease are plotted on the Y-axis against the corresponding S1 IgG binding titer for

657 each sample. Relative ranking of neutralization titers is indicated on the graph. Only sera that

658 gave a measurable titer in both semi-quantitative ELISA or pseudotype neutralization assay

659 were included in (B), (C) and (D). Serum sample groups are color-coded according to the

660 legend. (E) Concentrations of S1-specific serum IgG (pg) at ID $\mathrm{D}_{50}$ dilutions were calculated using

661 the IgG titers quantified via the semi-quantitative ELISA and the known ID $_{50}$ value. Only sera

662 that gave a measurable titer in both semi-quantitative ELISA and pseudotype neutralization

663 assay were included. Data for (A)-(B) and (E) were analyzed by a non-parametric Mann-

664 Whitney U test. 


\section{FIGURE 3}

A

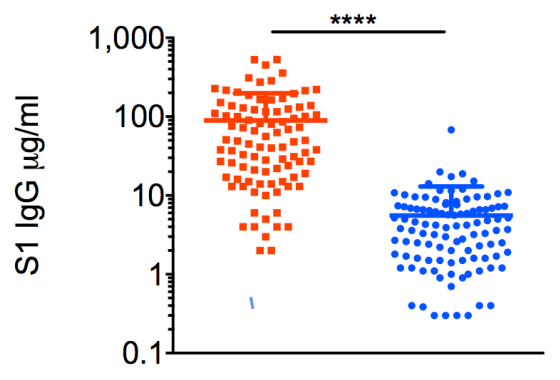

C

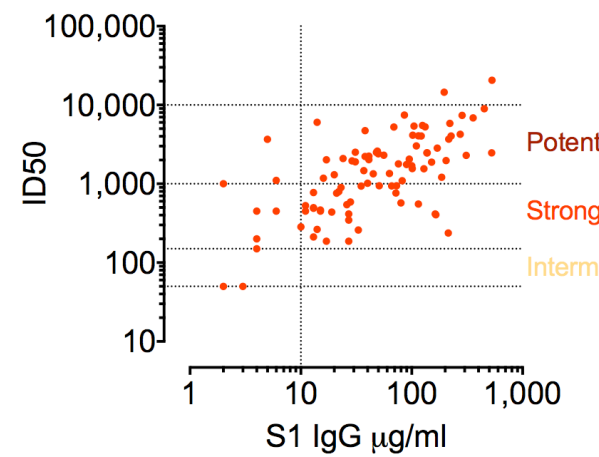

$\mathbf{E}$

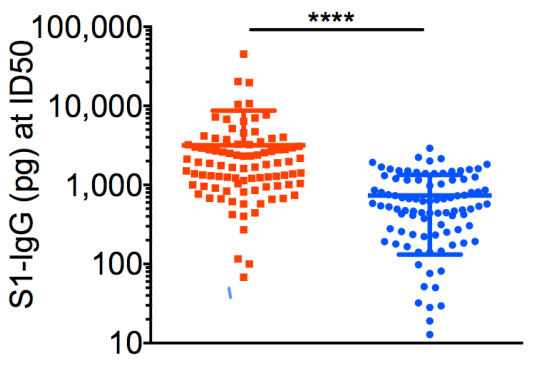

B

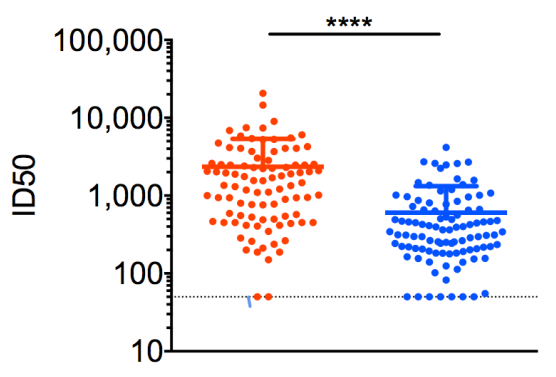

D

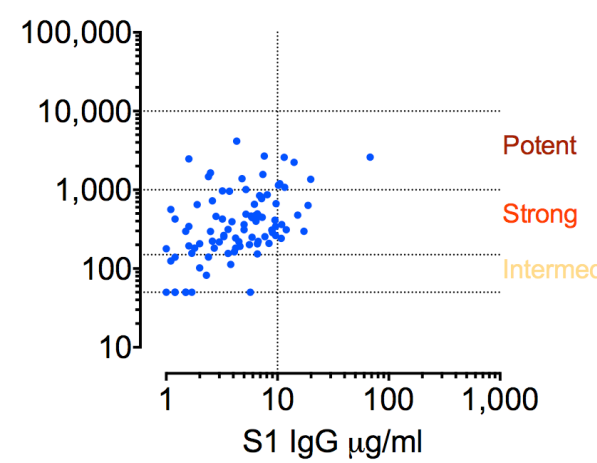

- Severe illness

- Mild illness 
666 Figure 4: Variant B.1.1.7 SARS-CoV-2 Spike effect on mAb and serum neutralization. (A)

667 Indicated mAbs were serially diluted in duplicate and incubated with the mutant SARS-CoV-2

668 luciferase-encoding pseudotyped virus in the legend prior to the addition of HeLa cells

669 expressing ACE-2. After two days neutralization was measured as the relative reduction in

670 relative light units (RLU). Data are representative of three independent repeats. The horizontal

671 dotted line on each graph indicates $50 \%$ neutralization. (B) $\mathrm{IC}_{50}$ values for each $\mathrm{mAb}$ or $\mathrm{ID}_{50}$

672 values for each serum sample against SARS-CoV-2 $\mathrm{D}_{614} \mathrm{G}$ pseudotyped virus were divided by the

$673 \mathrm{IC}_{50}$ for each mutant pseudotyped virus against the corresponding $\mathrm{mAb}$ to generate the fold

674 decrease in neutralization on the Y-axis, as color-coded in the key. The dotted horizontal line

675 indicates a 5-fold drop in neutralization potency. Whether fold decrease in neutralization

676 potency refers to mAbs, 18 serum samples from hospitalized patients or 18 serum samples

677 from healthcare workers who experience mild/asymptomatic COVID-19 is indicated under the

678 graph by the labels "mAbs", "severe illness" and "mild illness", respectively. 
bioRxiv preprint doi: https://doi.org/10.1101/2021.01.15.426849; this version posted January 19, 2021. The copyright holder for this preprint (which was not certified by peer review) is the author/funder, who has granted bioRxiv a license to display the preprint in perpetuity. It is made available under aCC-BY-ND 4.0 International license.

FIGURE 4
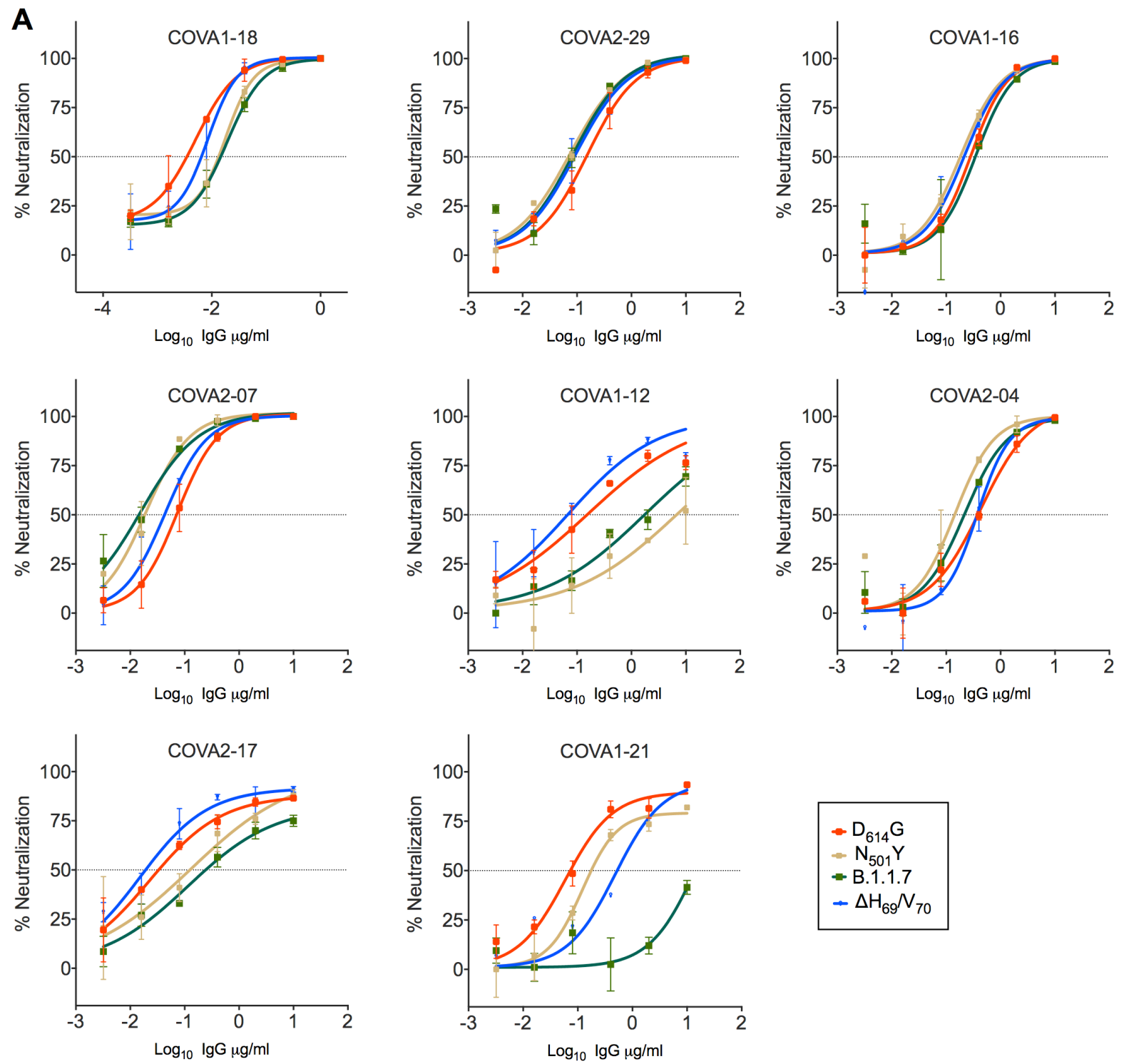

$$
\begin{aligned}
& -\mathrm{D}_{614} \mathrm{G} \\
& =\mathrm{N}_{501} \mathrm{Y} \\
& =\mathrm{B} .1 .1 .7 \\
& -\Delta \mathrm{H}_{69} N_{70}
\end{aligned}
$$

B

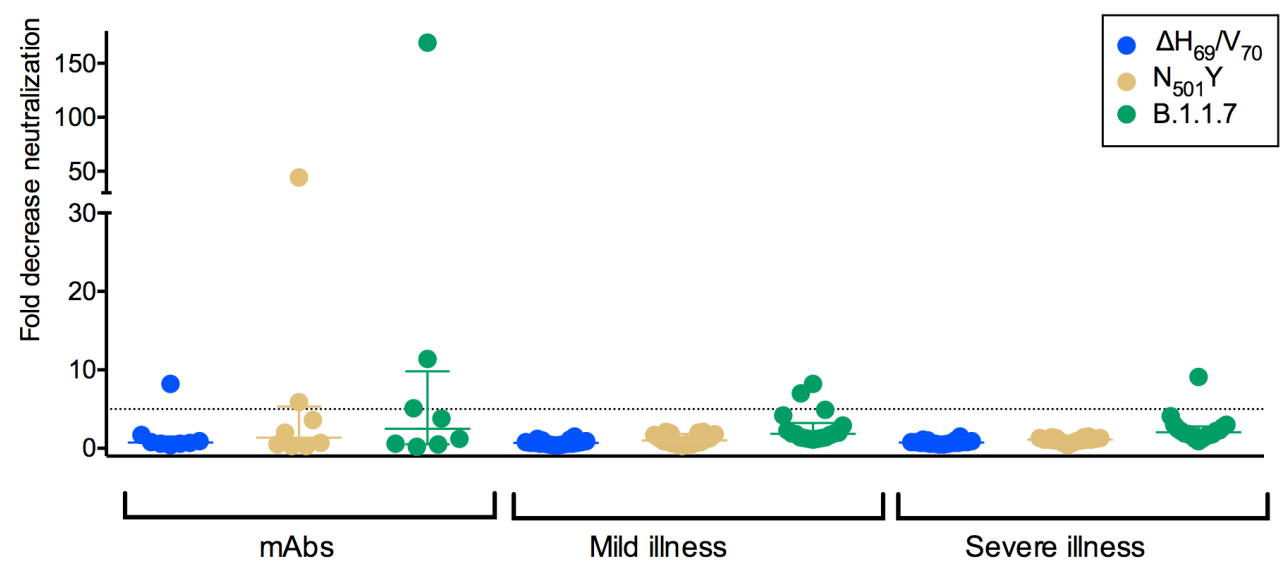

